Prepared for the U.S. Department of Energy under Contract DE-AC05-76RL01830

\title{
Analytical Data Report for Sediment Samples Collected From Well 299- E25-236 in the 200-PO-1 Operable Unit
}

Michael Lindberg

January 2009

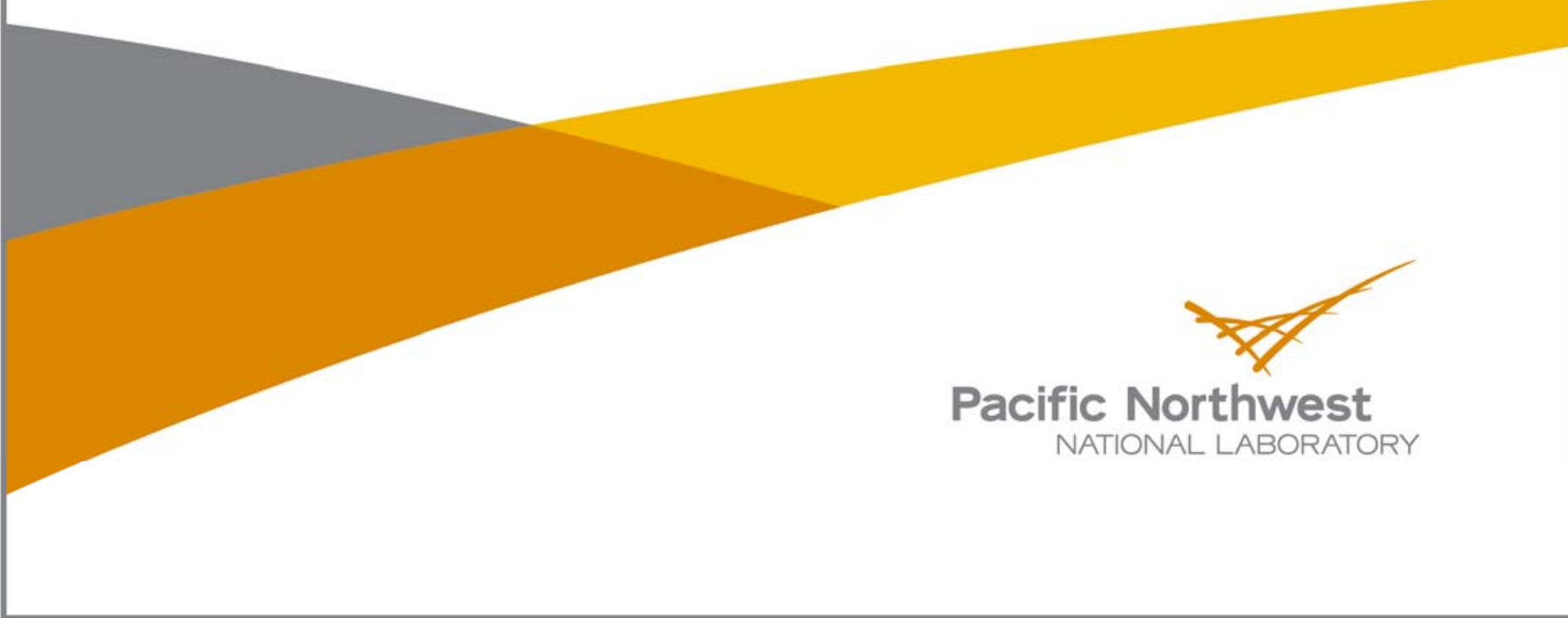




\title{
DISCLAIMER
}

This report was prepared as an account of work sponsored by an agency of the United States Government. Neither the United States Government nor any agency thereof, nor Battelle Memorial Institute, nor any of their employees, makes any warranty, express or implied, or assumes any legal liability or responsibility for the accuracy, completeness, or usefulness of any information, apparatus, product, or process disclosed, or represents that its use would not infringe privately owned rights. Reference herein to any specific commercial product, process, or service by trade name, trademark, manufacturer, or otherwise does not necessarily constitute or imply its endorsement, recommendation, or favoring by the United States Government or any agency thereof, or Battelle Memorial Institute. The views and opinions of authors expressed herein do not necessarily state or reflect those of the United States Government or any agency thereof.

\author{
PACIFIC NORTHWEST NATIONAL LABORATORY \\ operated by \\ BATTELLE \\ for the \\ UNITED STATES DEPARTMENT OF ENERGY \\ under Contract DE-AC05-76RL01830
}

Printed in the United States of America
Available to DOE and DOE contractors from the Office of Scientific and Technical Information,
P.O. Box 62, Oak Ridge, TN 37831-0062;
ph: (865) 576-8401
fax: $(865)$ 576-5728
email: reports@adonis.osti.gov

\begin{abstract}
Available to the public from the National Technical Information Service, U.S. Department of Commerce, 5285 Port Royal Rd., Springfield, VA 22161 ph: (800) 553-6847 fax: $(703) 605-6900$ email: orders@ntis.fedworld.gov online ordering: http://www.ntis.gov/ordering.htm
\end{abstract}

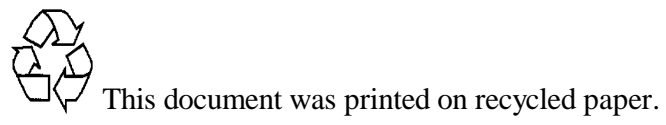




\section{Analytical Data Report for Sediment Samples Collected From Well 299- E25-236 in the 200-PO-1 Operable Unit}

M Lindberg

January 2009

Prepared for the U.S. Department of Energy under Contract DE-AC05-76RL01830

Pacific Northwest National Laboratory

Richland, Washington 99352 
01/27/09 15:24

To: Dale Dyekman

From: Michael J. Lindberg

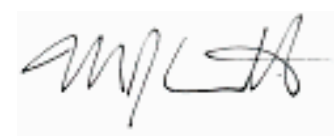

Environmental Sciences Laboratory

Energy and Environment Directorate, Pacific Northwest National Laboratory

Subject: Analytical Data Report for Sediment Samples Collected From Well 299-E25-236 in the 200-PO-1 Operable Unit, Sample Delivery Group ESL080031, SAF Number F08-150

This letter contains the following information for sample delivery group ESL080031

- Cover Sheet

- Narrative

- Analytical Results

- Quality Control

- Geologic Logs

- Geologic Photos

- Chain of Custodies 


\section{Introduction}

On September 11, 2008 sediment samples were received from Well 299-E25-236 in the 200-PO-1 Operable Unit for geochemical studies.

\section{Analytical Results/Methodology}

The analyses for this project were performed at the 325 building located in the 300 Area of the Hanford Site. The analyses were performed according to Pacific Northwest National Laboratory (PNNL) approved procedures and/or nationally recognized test procedures. The data sets include the sample identification numbers, analytical results, estimated quantification limits (EQL), and quality control data.

\section{Quality Control}

The preparatory and analytical quality control requirements, calibration requirements, acceptance criteria, and failure actions are defined in the on-line QA plan "Conducting Analytical Work in Support of Regulatory Programs" (CAW). This QA plan implements the Hanford Analytical Services Quality Assurance Requirements Documents (HASQARD) for PNNL.

\section{Definitions}

Dup Duplicate

RPD Relative Percent Difference

NR No Recovery (percent recovery less than zero)

ND Non-Detectable

$\%$ REC Percent Recovery

\section{Sample Receipt}

Samples were received with a chain of custody (COC) and were analyzed according to the sample identification numbers supplied by the client. All Samples were refrigerated upon receipt until prepared for analysis.

All samples were received with custody seals intact unless noted in the Case Narrative.

\section{Holding Times}

Holding time is defined as the time from sample preparation to the time of analyses. The prescribed holding times were met for all analytes unless noted in the Case Narrative.

\section{Analytical Results}

All reported analytical results meet the requirements of the CAW or client specified SOW unless noted in the case narrative. 


\section{Case Narrative Report}

\section{Hold Time:}

All samples were analyzed within the recommended holding times.

\section{Preparation Blank (PB):}

No discrepancies noted.

\section{Duplicate (DUP):}

No discrepancies noted.

\section{Laboratory Control Samples (LCS):}

An LCS was not run for alkalinity, specific conductivity and $\mathrm{pH}$ for these samples. There is no commercially available material to monitor preparation effectiveness for these constituents, so a blank spike would be utilized. For this process the blank spike would be the same as the standard used for the calibration or calibration checks. There would no difference between the ICV and an LCS with the exception of filtering the sample through a $0.45 \mathrm{uM}$ filter. The filtering process would not impact the calibration or calibration check solution concentrations. Therefore the ICV should be considered an LCS for these constituents.

The samples analyzed for this project were considered non-radiological. In order to run an LCS for Tc-99, the samples would have to be prepared in a radiological area. The samples and associated liquids prepared in a radiologically controlled area would be considered radiological. To maintain ALARA principals and to reduce the amount of radioactive waste produced, a radiological LCS is typically not performed. There should be no impact to data as reported since the same preparation for total and trace metals is used for radionuclides. The extraction efficiencies were monitored by the LCS for the total and trace metals.

The blank spike solution analyzed as the LCS for inductively coupled plasma optical emissions spectrometry does not contain strontium. Preparation effectiveness for this constituent is monitored by the recoveries of the other analytes contained in the blank spike. There should be no impact to the data as reported.

\section{Post Spike (PS):}

Post-Spike Recovery for Sulfate (45.1\%) was outside acceptance limits (75-125) in 8I24001-PS1 for Anions by IC-WE The native sample concentration was greater than 5 times the spiked concentration.

Post-Spike Recovery for Calcium (221\%) was outside acceptance limits (75-125) in 8I26001-PS1 for ICP-OES Vadose-WE The native sample concentration was greater than 5 times the spiked concentration.

Post-Spike Recovery for Magnesium (72.4\%) was outside acceptance limits (75-125) in 8I26001-PS1 for ICP-OES Vadose-WE The native sample concentration was greater than 5 times the spiked concentration.

Post-Spike Recovery for Sodium (150\%) was outside acceptance limits (75-125) in 8I26001-PS1 for ICP-OES Vadose-WE The native sample concentration was greater than 5 times the spiked concentration.

\section{Matrix Spike (MS):}

Not Applicable

\section{Other QC Criteria:}

No discrepancies noted. 


\section{DISCLAIMER}

This report was prepared as an account of work sponsored by an agency of the United States Government. Neither the United States Government nor any agency thereof, nor Battelle Memorial Institute, nor any of their employees, makes any warranty, express or implied, or assumes any legal liability or responsibility for the accuracy, completeness, or usefulness of any information, apparatus, product, or process disclosed, or represents that its use would not infringe privately owned rights. Reference herein to any specific commercial product, process, or service by trade name, trademark, manufacturer, or otherwise does not necessarily constitute or imply its endorsement, recommendation, or favoring by the United States Government or any agency thereof, or Battelle Memorial Institute. The views and opinions of authors expressed herein do not necessarily state or reflect those of the United States Government or any agency thereof.

\section{SAMPLES INCLUDED IN THIS REPORT}

\section{A-AX, C6542}

HEIS No.

B1WPB6

B1WPB7

B1WPB8

B1WPB9

$\begin{array}{ll}\text { Laboratory ID } & \text { Matrix } \\ 0809002-01 & \text { SOIL } \\ 0809002-02 & \text { SOIL } \\ 0809002-03 & \text { SOIL } \\ 0809002-04 & \text { SOIL }\end{array}$

$\begin{array}{ll}\text { Date Collected } \\ \text { 9/8/08 } & 10: 10 \\ 9 / 8 / 08 & 10: 40 \\ 9 / 9 / 08 & 07: 45 \\ 9 / 9 / 08 & 08: 30\end{array}$

Date Received

9/11/08 10:05

9/11/08 10:05

9/11/08 10:05

9/11/08 10:05 
The following analyses were performed on the following samples included in this report:

Metals 1:1 DI Water Extract by ICPMS

Metals 1:1 Water Extract by ICPOES

Alkalinity, Titrimetic ( $\mathrm{pH} 4.5)$

Anions By Ion Chromatography

Geological Description

Moisture Content

$\mathrm{pH}$ of Waters By Electrode

Specific Conductance

Tc_U 1:1 DI Water Extract by ICPMS

\section{SAMPLES ANALYZED IN THIS REPORT}

$\begin{array}{lll}\text { HEIS No. } & \text { Laboratory ID } & \text { Matrix } \\ \text { B1WPB6 } & 0809002-01 & \text { SOIL } \\ \text { B1WPB7 } & 0809002-02 & \text { SOIL } \\ \text { B1WPB8 } & 0809002-03 & \text { SOIL } \\ \text { B1WPB9 } & 0809002-04 & \text { SOIL }\end{array}$

Date Collected
$\begin{array}{ll}9 / 8 / 08 & 10: 10 \\ 9 / 8 / 08 & 10: 40 \\ 9 / 9 / 08 & 07: 45 \\ 9 / 9 / 08 & 08: 30\end{array}$

Date Received 9/11/08 10:05

9/11/08 10:05

9/11/08 10:05

9/11/08 10:05 


\section{Wet Chemistry}

Alkalinity as CaCO3 (ug/g dry) by Standard Methods 2320B

Lab ID HEIS No.

Results

EQL

Analyzed

Batch

0809002-01

B1WPB6

3.05E1

2.34E1

$10 / 01 / 08$

$8 \mathrm{I} 29002$

0809002-02

B1WPB7

3.37E1

$2.35 \mathrm{E} 1$

$10 / 01 / 08$

$8 \mathrm{I} 29002$

0809002-03

B1WPB8

3.43E1

$2.35 \mathrm{E} 1$

$10 / 01 / 08$

$8 \mathrm{I} 29002$

0809002-04 B1WPB9

4.82E1 2.35E1

$10 / 01 / 08$

$8 \mathrm{I} 29002$ 


\section{Wet Chemistry}

Specific Conductance (EC) (mS/cm) by EPA 120.1

\begin{tabular}{llcccc} 
Lab ID & HEIS No. & Results & EQL & Analyzed & Batch \\
\hline $0809002-01$ & B1WPB6 & $2.61 \mathrm{E}-1$ & $1.00 \mathrm{E}-2$ & $9 / 24 / 08$ & $8 \mathrm{I} 23003$ \\
$0809002-02$ & B1WPB7 & $2.82 \mathrm{E}-1$ & $1.00 \mathrm{E}-2$ & $9 / 24 / 08$ & $8 \mathrm{I} 23003$ \\
$0809002-03$ & B1WPB8 & $4.99 \mathrm{E}-1$ & $1.00 \mathrm{E}-2$ & $9 / 24 / 08$ & $8 \mathrm{I} 23003$ \\
$0809002-04$ & B1WPB9 & $9.90 \mathrm{E}-1$ & $1.00 \mathrm{E}-2$ & $9 / 24 / 08$ & $8 \mathrm{I} 23003$ \\
\hline
\end{tabular}




\section{Wet Chemistry}

Moisture Content (\% by Weight) by AGG-WC-001

Lab ID HEIS No. Results

0809002-01 B1WPB6

4.31E0

EQL

Analyzed

Batch

0809002-02

B1WPB7

$4.83 \mathrm{E} 0$

N/A

9/19/08

$8 \mathrm{I} 11007$

0809002-03

B1WPB8

$1.16 \mathrm{E} 1$

N/A

9/19/08

$8 \mathrm{I} 11007$

0809002-04 B1WPB9

$2.33 \mathrm{E} 1$

$\mathrm{N} / \mathrm{A}$

9/19/08

$8 \mathrm{I} 11007$

$9 / 19 / 08$

$8 \mathrm{I} 11007$ 


\section{Wet Chemistry}

pH (pH Units) by AGG-pH-001

Lab HEIS No.

Results

EQL

Analyzed

Batch

0809002-01 B1WPB6

7.95E0

N/A

9/24/08

$8 \mathrm{I} 23002$

0809002-02

B1WPB7

$7.81 \mathrm{E} 0$

N/A

$9 / 24 / 08$

$8 \mathrm{I} 23002$

0809002-03

B1WPB8

7.64E0

N/A

$9 / 24 / 08$

$8 \mathrm{I} 23002$

0809002-04 B1WPB9

$7.72 \mathrm{E} 0$

N/A

$9 / 24 / 08$

$8 \mathrm{I} 23002$ 


\section{Anions by Ion Chromatography}

\begin{tabular}{|c|c|c|c|c|c|c|c|}
\hline CAS \# & Analyte & Results & Units & EQL & Analyzed & Batch & Method \\
\hline HEIS No. & B1WPB6 & \multicolumn{3}{|c|}{ Lab ID: $\quad$ 0809002-01 } & & & \\
\hline $16984-48-8$ & Fluoride & $2.49 \mathrm{E}-1$ & ug/g dry & $1.99 \mathrm{E}-1$ & $9 / 24 / 08$ & $8 \mathrm{I} 24001$ & AGG-IC-001 \\
\hline $16887-00-6$ & Chloride & $1.96 \mathrm{E} 0$ & ug/g dry & $4.99 \mathrm{E}-1$ & $9 / 24 / 08$ & $8 \mathrm{I} 24001$ & AGG-IC-001 \\
\hline $14797-55-8$ & Nitrate & $1.23 \mathrm{E} 1$ & ug/g dry & $9.97 \mathrm{E}-1$ & $9 / 24 / 08$ & $8 \mathrm{I} 24001$ & AGG-IC-001 \\
\hline $14808-79-8$ & Sulfate & $8.27 \mathrm{E} 1$ & ug/g dry & $1.50 \mathrm{E} 0$ & $9 / 24 / 08$ & $8 \mathrm{I} 24001$ & AGG-IC-001 \\
\hline $14265-44-2$ & Phosphate & $<1.50 \mathrm{E} 0$ & ug/g dry & $1.50 \mathrm{E} 0$ & $9 / 24 / 08$ & $8 \mathrm{I} 24001$ & AGG-IC-001 \\
\hline HEIS No. & B1WPB7 & \multicolumn{3}{|c|}{ Lab ID: $\quad$ 0809002-02 } & & & \\
\hline $16984-48-8$ & Fluoride & $2.98 \mathrm{E}-1$ & ug/g dry & $2.00 \mathrm{E}-1$ & $9 / 24 / 08$ & $8 \mathrm{I} 24001$ & AGG-IC-001 \\
\hline $16887-00-6$ & Chloride & $2.11 \mathrm{E} 0$ & ug/g dry & $5.01 \mathrm{E}-1$ & $9 / 24 / 08$ & $8 \mathrm{I} 24001$ & AGG-IC-001 \\
\hline $14797-55-8$ & Nitrate & $1.22 \mathrm{E} 1$ & ug/g dry & $1.00 \mathrm{E} 0$ & $9 / 24 / 08$ & $8 \mathrm{I} 24001$ & AGG-IC-001 \\
\hline $14808-79-8$ & Sulfate & $9.36 \mathrm{E} 1$ & ug/g dry & $1.50 \mathrm{E} 0$ & $9 / 24 / 08$ & $8 \mathrm{I} 24001$ & AGG-IC-001 \\
\hline $14265-44-2$ & Phosphate & $<1.50 \mathrm{E} 0$ & ug/g dry & $1.50 \mathrm{E} 0$ & $9 / 24 / 08$ & $8 \mathrm{I} 24001$ & AGG-IC-001 \\
\hline HEIS No. & B1WPB8 & \multicolumn{3}{|c|}{ Lab ID: $\quad$ 0809002-03 } & & & \\
\hline $16984-48-8$ & Fluoride & $2.76 \mathrm{E}-1$ & ug/g dry & $2.00 \mathrm{E}-1$ & $9 / 24 / 08$ & $8 \mathrm{I} 24001$ & AGG-IC-001 \\
\hline $16887-00-6$ & Chloride & $5.00 \mathrm{E} 0$ & ug/g dry & $4.99 \mathrm{E}-1$ & $9 / 24 / 08$ & $8 \mathrm{I} 24001$ & AGG-IC-001 \\
\hline $14797-55-8$ & Nitrate & $2.87 \mathrm{E} 1$ & ug/g dry & $9.98 \mathrm{E}-1$ & $9 / 24 / 08$ & $8 \mathrm{I} 24001$ & AGG-IC-001 \\
\hline $14808-79-8$ & Sulfate & $2.03 \mathrm{E} 2$ & ug/g dry & $1.50 \mathrm{E} 1$ & $9 / 25 / 08$ & $8 \mathrm{I} 24001$ & AGG-IC-001 \\
\hline $14265-44-2$ & Phosphate & $<1.50 \mathrm{E} 0$ & ug/g dry & $1.50 \mathrm{E} 0$ & $9 / 24 / 08$ & $8 \mathrm{I} 24001$ & AGG-IC-001 \\
\hline HEIS No. & B1WPB9 & \multicolumn{3}{|c|}{ Lab ID: $\quad$ 0809002-04 } & & & \\
\hline $16984-48-8$ & Fluoride & $<2.00 \mathrm{E} 0$ & ug/g dry & $2.00 \mathrm{E} 0$ & $9 / 24 / 08$ & $8 \mathrm{I} 24001$ & AGG-IC-001 \\
\hline $16887-00-6$ & Chloride & $1.00 \mathrm{E} 1$ & ug/g dry & $5.00 \mathrm{E} 0$ & $9 / 24 / 08$ & $8 \mathrm{I} 24001$ & AGG-IC-001 \\
\hline $14797-55-8$ & Nitrate & $6.12 \mathrm{E} 1$ & ug/g dry & $1.00 \mathrm{E} 1$ & $9 / 24 / 08$ & $8 \mathrm{I} 24001$ & AGG-IC-001 \\
\hline $14808-79-8$ & Sulfate & $4.70 \mathrm{E} 2$ & ug/g dry & $1.50 \mathrm{E} 1$ & $9 / 24 / 08$ & $8 \mathrm{I} 24001$ & AGG-IC-001 \\
\hline $14265-44-2$ & Phosphate & $<1.50 \mathrm{E} 1$ & ug/g dry & $1.50 \mathrm{E} 1$ & $9 / 24 / 08$ & $8 \mathrm{I} 24001$ & AGG-IC-001 \\
\hline
\end{tabular}


Total Metals by PNNL-AGG-ICP-AES/Water Extract

\begin{tabular}{|c|c|c|c|c|c|c|c|}
\hline CAS \# & Analyte & Results & Units & EQL & Analyzed & Batch & Method \\
\hline HEIS No. & B1WPB6 & \multicolumn{3}{|c|}{ Lab ID: $\quad$ 0809002-01 } & & & \\
\hline $7440-70-2$ & Calcium & $2.56 \mathrm{E} 1$ & $u g / g$ dry & $3.86 \mathrm{E}-1$ & $9 / 26 / 08$ & $8 \mathrm{I} 26001$ & PNNL-AGG-ICP-AES \\
\hline $7440-09-7$ & Potassium & $3.16 \mathrm{E} 0$ & $\mathrm{ug} / \mathrm{g}$ dry & $2.32 \mathrm{E} 0$ & $9 / 26 / 08$ & $8 \mathrm{I} 26001$ & PNNL-AGG-ICP-AES \\
\hline $7439-95-4$ & Magnesium & $5.44 \mathrm{E} 0$ & ug/g dry & $8.32 \mathrm{E}-2$ & $9 / 26 / 08$ & $8 \mathrm{I} 26001$ & PNNL-AGG-ICP-AES \\
\hline $7440-24-6$ & Strontium & $9.64 \mathrm{E}-2$ & ug/g dry & $5.21 \mathrm{E}-2$ & $9 / 26 / 08$ & $8 \mathrm{I} 26001$ & PNNL-AGG-ICP-AES \\
\hline $7440-23-5$ & Sodium & $1.49 \mathrm{E} 1$ & ug/g dry & $6.67 \mathrm{E}-1$ & $9 / 26 / 08$ & $8 \mathrm{I} 26001$ & PNNL-AGG-ICP-AES \\
\hline HEIS No. & B1WPB7 & \multicolumn{3}{|c|}{ Lab ID: $\quad$ 0809002-02 } & & & \\
\hline $7440-70-2$ & Calcium & $2.87 \mathrm{E} 1$ & ug/g dry & $3.87 \mathrm{E}-1$ & $9 / 26 / 08$ & $8 \mathrm{I} 26001$ & PNNL-AGG-ICP-AES \\
\hline $7440-09-7$ & Potassium & 3.67E0 & ug/g dry & $2.33 \mathrm{E} 0$ & $9 / 26 / 08$ & $8 \mathrm{I} 26001$ & PNNL-AGG-ICP-AES \\
\hline $7439-95-4$ & Magnesium & 5.84E0 & ug/g dry & $8.35 \mathrm{E}-2$ & $9 / 26 / 08$ & $8 \mathrm{I} 26001$ & PNNL-AGG-ICP-AES \\
\hline $7440-24-6$ & Strontium & $1.15 \mathrm{E}-1$ & ug/g dry & $5.23 \mathrm{E}-2$ & $9 / 26 / 08$ & $8 \mathrm{I} 26001$ & PNNL-AGG-ICP-AES \\
\hline $7440-23-5$ & Sodium & $1.68 \mathrm{E} 1$ & ug/g dry & $6.70 \mathrm{E}-1$ & $9 / 26 / 08$ & $8 \mathrm{I} 26001$ & PNNL-AGG-ICP-AES \\
\hline HEIS No. & B1WPB8 & \multicolumn{3}{|c|}{ Lab ID: $\quad$ 0809002-03 } & & & \\
\hline $7440-70-2$ & Calcium & $5.88 \mathrm{E} 1$ & ug/g dry & $3.86 \mathrm{E}-1$ & $9 / 26 / 08$ & $8 \mathrm{I} 26001$ & PNNL-AGG-ICP-AES \\
\hline $7440-09-7$ & Potassium & 4.79E0 & ug/g dry & $2.32 \mathrm{E} 0$ & $9 / 26 / 08$ & $8 \mathrm{I} 26001$ & PNNL-AGG-ICP-AES \\
\hline $7439-95-4$ & Magnesium & $1.44 \mathrm{E} 1$ & ug/g dry & $8.33 \mathrm{E}-2$ & $9 / 26 / 08$ & $8 \mathrm{I} 26001$ & PNNL-AGG-ICP-AES \\
\hline $7440-24-6$ & Strontium & $2.85 \mathrm{E}-1$ & ug/g dry & $5.21 \mathrm{E}-2$ & $9 / 26 / 08$ & $8 \mathrm{I} 26001$ & PNNL-AGG-ICP-AES \\
\hline $7440-23-5$ & Sodium & $2.58 \mathrm{E} 1$ & $\mathrm{ug} / \mathrm{g}$ dry & $6.68 \mathrm{E}-1$ & $9 / 26 / 08$ & $8 \mathrm{I} 26001$ & PNNL-AGG-ICP-AES \\
\hline HEIS No. & B1WPB9 & \multicolumn{3}{|c|}{ Lab ID: $\quad$ 0809002-04 } & & & \\
\hline $7440-70-2$ & Calcium & $1.25 \mathrm{E} 2$ & ug/g dry & $3.87 \mathrm{E}-1$ & $9 / 26 / 08$ & $8 \mathrm{I} 26001$ & PNNL-AGG-ICP-AES \\
\hline $7440-09-7$ & Potassium & 7.19E0 & ug/g dry & $2.33 \mathrm{E} 0$ & $9 / 26 / 08$ & $8 \mathrm{I} 26001$ & PNNL-AGG-ICP-AES \\
\hline $7439-95-4$ & Magnesium & 3.62E1 & ug/g dry & $8.35 \mathrm{E}-2$ & $9 / 26 / 08$ & $8 \mathrm{I} 26001$ & PNNL-AGG-ICP-AES \\
\hline $7440-24-6$ & Strontium & $6.00 \mathrm{E}-1$ & ug/g dry & $5.22 \mathrm{E}-2$ & $9 / 26 / 08$ & $8 \mathrm{I} 26001$ & PNNL-AGG-ICP-AES \\
\hline $7440-23-5$ & Sodium & $5.28 \mathrm{E} 1$ & ug/g dry & $6.70 \mathrm{E}-1$ & $9 / 26 / 08$ & $8 \mathrm{I} 26001$ & PNNL-AGG-ICP-AES \\
\hline
\end{tabular}




\section{Radionuclides by ICP-MS/Water Extract}

\begin{tabular}{|c|c|c|c|c|c|c|c|}
\hline CAS \# & Analyte & Results & Units & EQL & Analyzed & Batch & Method \\
\hline HEIS No. & B1WPB6 & \multicolumn{3}{|c|}{ Lab ID: $\quad 0809002-01$} & & & \\
\hline $14133-76-7$ & Technetium-99 & $<2.29 \mathrm{E}-5$ & ug/g dry & $2.29 \mathrm{E}-5$ & $9 / 26 / 08$ & $8 \mathrm{I} 26004$ & PNNL-AGG-415 \\
\hline HEIS No. & B1WPB7 & \multicolumn{3}{|c|}{ Lab ID: $\quad 0809002-02$} & & & \\
\hline $14133-76-7$ & Technetium-99 & $<2.30 \mathrm{E}-5$ & ug/g dry & $2.30 \mathrm{E}-5$ & $9 / 26 / 08$ & $8 \mathrm{I} 26004$ & PNNL-AGG-415 \\
\hline HEIS No. & B1WPB8 & \multicolumn{3}{|c|}{ Lab ID: $\quad 0809002-03$} & & & \\
\hline $14133-76-7$ & Technetium-99 & $<2.30 \mathrm{E}-5$ & ug/g dry & $2.30 \mathrm{E}-5$ & $9 / 26 / 08$ & $8 \mathrm{I} 26004$ & PNNL-AGG-415 \\
\hline HEIS No. & B1WPB9 & \multicolumn{3}{|c|}{ Lab ID: $\quad$ 0809002-04 } & & & \\
\hline $14133-76-7$ & Technetium-99 & $<2.30 \mathrm{E}-5$ & ug/g dry & $2.30 \mathrm{E}-5$ & $9 / 26 / 08$ & $8 \mathrm{I} 26004$ & PNNL-AGG-415 \\
\hline
\end{tabular}


RCRA Metals By PNNL-AGG-415/Water Extract

\begin{tabular}{|c|c|c|c|c|c|c|c|}
\hline CAS \# & Analyte & Results & Units & EQL & Analyzed & Batch & Method \\
\hline HEIS No. & B1WPB6 & \multicolumn{3}{|c|}{ Lab ID: $\quad$ 0809002-01 } & & & \\
\hline $13966-28-4$ & Lead & $<5.59 \mathrm{E}-4$ & ug/g dry & $5.59 \mathrm{E}-4$ & $9 / 26 / 08$ & $8 \mathrm{I} 26002$ & PNNL-AGG-415 \\
\hline HEIS No. & B1WPB7 & \multicolumn{3}{|c|}{ Lab ID: $\quad$ 0809002-02 } & & & \\
\hline $13966-28-4$ & Lead & $<5.61 \mathrm{E}-4$ & ug/g dry & $5.61 \mathrm{E}-4$ & $9 / 26 / 08$ & $8 \mathrm{I} 26002$ & PNNL-AGG-415 \\
\hline HEIS No. & B1WPB8 & \multicolumn{3}{|c|}{ Lab ID: $\quad 0809002-03$} & & & \\
\hline $13966-28-4$ & Lead & $<5.59 \mathrm{E}-4$ & ug/g dry & $5.59 \mathrm{E}-4$ & $9 / 26 / 08$ & $8 \mathrm{I} 26002$ & PNNL-AGG-415 \\
\hline HEIS No. & B1WPB9 & \multicolumn{3}{|c|}{ Lab ID: $\quad 0809002-04$} & & & \\
\hline $13966-28-4$ & Lead & $<5.60 \mathrm{E}-4$ & ug/g dry & $5.60 \mathrm{E}-4$ & $9 / 26 / 08$ & $8 \mathrm{I} 26002$ & PNNL-AGG-415 \\
\hline
\end{tabular}


Wet Chemistry - Quality Control

Environmental Science Laboratory

\begin{tabular}{|c|c|c|c|c|c|c|c|c|c|c|}
\hline Analyte & Result & $\begin{array}{c}\text { orting } \\
\text { Limit }\end{array}$ & Units & $\begin{array}{l}\text { Spike } \\
\text { Level }\end{array}$ & $\begin{array}{l}\text { Source } \\
\text { Result }\end{array}$ & $\%$ REC & $\begin{array}{l}\text { \%REC } \\
\text { Limits }\end{array}$ & RPD & $\begin{array}{l}\text { RPD } \\
\text { Limit }\end{array}$ & Notes \\
\hline
\end{tabular}

Batch 8I23002 - 1:1 Water Extract (pH_EC_Alk)

Duplicate (8I23002-DUP1)

$\mathrm{pH}$

Source: 0809002-04

Prepared: 09/23/08 Analyzed: 09/24/08

7.70E0 N/A pH Units

$7.72 \mathrm{E} 0$

0.259

35

Batch 8I23003 - 1:1 Water Extract (pH_EC_Alk)

Blank (8I23003-BLK1)

Specific Conductance (EC)

$<1.00 \mathrm{E}-2 \quad 1.00 \mathrm{E}-2 \quad \mathrm{mS} / \mathrm{cm}$

Prepared: 09/23/08 Analyzed: 09/24/08

Duplicate (8I23003-DUP1)

Source: 0809002-04

Prepared: 09/23/08 Analyzed: 09/24/08

Specific Conductance (EC)

$1.02 \mathrm{E} 0 \quad 1.00 \mathrm{E}-2 \quad \mathrm{mS} / \mathrm{cm}$

$9.90 \mathrm{E}-1$

2.59

35

Batch 8I29002 - 1:1 Water Extract (pH_EC_Alk)

Blank (8I29002-BLK1)

Prepared: 09/29/08 Analyzed: 10/01/08

Alkalinity as $\mathrm{CaCO} 3$

$<2.35 \mathrm{E} 1$

$2.35 \mathrm{E} 1 \mathrm{ug} / \mathrm{g}$ wet

Duplicate (8I29002-DUP1)

Source: 0809002-04 Prepared: 09/29/08 Analyzed: 10/01/08

Alkalinity as $\mathrm{CaCO} 3$

4.90E1 2.35E1 ug/g dry

$4.82 \mathrm{E} 1$

$1.62 \quad 35$ 
Anions by Ion Chromatography - Quality Control

Environmental Science Laboratory

\begin{tabular}{|lrrrrrrrrrrr}
\hline & & Reporting & & Spike & Source & & & $\%$ REC & & RPD \\
Analyte & Result & Limit & Units & Level & Result & $\%$ REC & Limits & RPD & Limit & Notes \\
\hline
\end{tabular}

Batch 8I24001 - 1:1 Water Extract (IC)

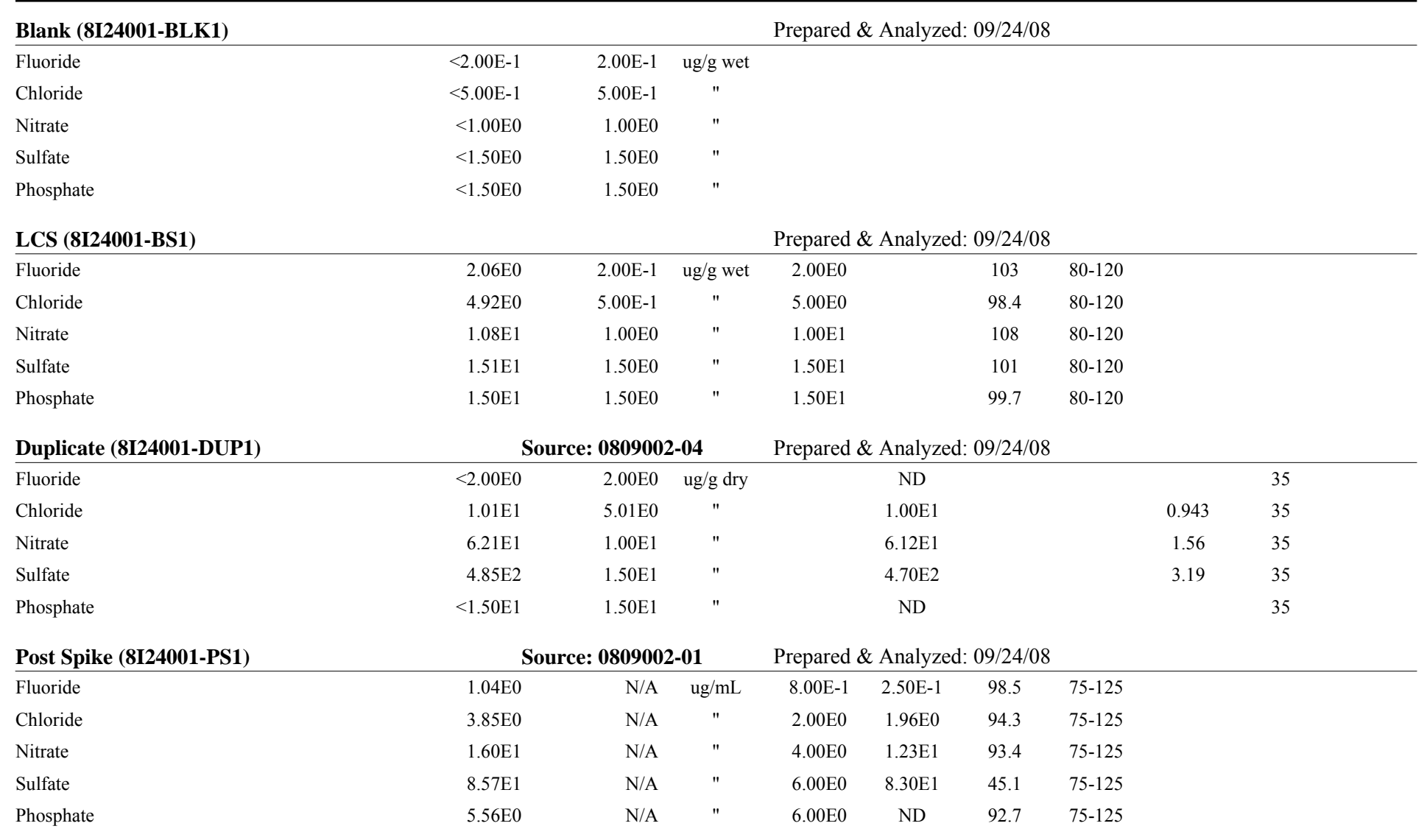


Total Metals by PNNL-AGG-ICP-AES/Water Extract - Quality Control

Environmental Science Laboratory

\begin{tabular}{|lrrrrrrrrrr}
\hline & & Reporting & & Spike & Source & & & $\%$ REC & & RPD \\
Analyte & Result & Limit & Units & Level & Result & $\%$ REC & Limits & RPD & Limit & Notes \\
\hline
\end{tabular}

Batch 8I26001 - 1:1 Water Extract (ICP/ICPMS)

\begin{tabular}{|c|c|c|c|c|c|c|c|c|c|}
\hline \multirow{2}{*}{$\begin{array}{l}\text { Blank (8I26001-BLK1) } \\
\text { Calcium }\end{array}$} & \multicolumn{9}{|c|}{ Prepared: 09/23/08 Analyzed: 09/26/08 } \\
\hline & $<3.87 \mathrm{E}-1$ & $3.87 \mathrm{E}-1$ & $\mathrm{ug} / \mathrm{g}$ wet & & & & & & \\
\hline Potassium & $<2.33 \mathrm{E} 0$ & 2.33E0 & $"$ & & & & & & \\
\hline Magnesium & $<8.34 \mathrm{E}-2$ & $8.34 \mathrm{E}-2$ & $"$ & & & & & & \\
\hline Strontium & $<5.22 \mathrm{E}-2$ & $5.22 \mathrm{E}-2$ & $"$ & & & & & & \\
\hline Sodium & $<6.69 \mathrm{E}-1$ & $6.69 \mathrm{E}-1$ & $"$ & & & & & & \\
\hline LCS (8I26001-BS1) & \multicolumn{9}{|c|}{ Prepared: 09/23/08 Analyzed: 09/26/08 } \\
\hline Calcium & 4.79E0 & $3.87 \mathrm{E}-1$ & ug/g wet & $4.99 \mathrm{E} 0$ & & 96.0 & $80-120$ & & \\
\hline Potassium & $4.95 \mathrm{E} 1$ & $2.33 \mathrm{E} 0$ & $"$ & 4.99E1 & & 99.1 & $80-120$ & & \\
\hline Magnesium & $4.86 \mathrm{E} 0$ & $8.34 \mathrm{E}-2$ & $"$ & $4.99 \mathrm{E} 0$ & & 97.4 & $80-120$ & & \\
\hline Strontium & NA & NA & $"$ & & & & $80-120$ & & \\
\hline Sodium & $5.16 \mathrm{E} 0$ & $6.69 \mathrm{E}-1$ & $"$ & $4.99 \mathrm{E} 0$ & & 103 & $80-120$ & & \\
\hline Duplicate (8I26001-DUP1) & \multicolumn{3}{|c|}{ Source: 0809002-04 } & \multicolumn{6}{|c|}{ Prepared: 09/23/08 Analyzed: 09/26/08 } \\
\hline Calcium & $1.30 \mathrm{E} 2$ & $3.88 \mathrm{E}-1$ & $\mathrm{ug} / \mathrm{g}$ dry & & $1.25 \mathrm{E} 2$ & & & 4.24 & 35 \\
\hline Potassium & $7.17 \mathrm{E} 0$ & $2.33 \mathrm{E} 0$ & $"$ & & 7.19E0 & & & 0.290 & 35 \\
\hline Magnesium & $3.69 \mathrm{E} 1$ & $8.35 \mathrm{E}-2$ & $"$ & & $3.62 \mathrm{E} 1$ & & & 2.10 & 35 \\
\hline Strontium & $6.17 \mathrm{E}-1$ & $5.23 \mathrm{E}-2$ & $"$ & & $6.00 \mathrm{E}-1$ & & & 2.78 & 35 \\
\hline Sodium & $5.41 \mathrm{E} 1$ & $6.70 \mathrm{E}-1$ & $"$ & & $5.28 \mathrm{E} 1$ & & & 2.32 & 35 \\
\hline Post Spike (8I26001-PS1) & \multicolumn{3}{|c|}{ Source: 0809002-04 } & \multicolumn{4}{|c|}{ Prepared \& Analyzed: 09/26/08 } & & \\
\hline Calcium & $4.26 \mathrm{E} 4$ & $\mathrm{~N} / \mathrm{A}$ & $\mathrm{ug} / \mathrm{L}$ & $5.00 \mathrm{E} 2$ & $4.15 \mathrm{E} 4$ & 221 & $75-125$ & & \\
\hline Potassium & $3.68 \mathrm{E} 3$ & N/A & $"$ & $1.25 \mathrm{E} 3$ & $2.39 \mathrm{E} 3$ & 103 & $75-125$ & & \\
\hline Magnesium & $1.24 \mathrm{E} 4$ & N/A & $"$ & $5.00 \mathrm{E} 2$ & $1.20 \mathrm{E} 4$ & 72.4 & $75-125$ & & \\
\hline Strontium & $6.95 \mathrm{E} 2$ & N/A & $"$ & $5.00 \mathrm{E} 2$ & $2.00 \mathrm{E} 2$ & 99 & $75-125$ & & \\
\hline Sodium & $1.83 \mathrm{E} 4$ & N/A & $"$ & $5.00 \mathrm{E} 2$ & $1.76 \mathrm{E} 4$ & 150 & $75-125$ & & \\
\hline
\end{tabular}




\section{Radionuclides by ICP-MS/Water Extract - Quality Control \\ Environmental Science Laboratory}

\begin{tabular}{|c|c|c|c|c|c|c|c|c|c|c|}
\hline & & orting & & Spike & Source & & $\%$ REC & & RPD & \\
\hline Analyte & Result & Limit & Units & Level & Result & $\%$ REC & Limits & RPD & Limit & Notes \\
\hline
\end{tabular}

Batch 8I26004 - 1:1 Water Extract (ICP/ICPMS)

Blank (8I26004-BLK1)

Technetium-99

Duplicate (8I26004-DUP1)

Technetium-99

Post Spike (8I26004-PS1)

Technetium-99
Prepared \& Analyzed: 09/26/08

Prepared \& Analyzed: 09/26/08

ND

35

Source: 0809002-04 Prepared \& Analyzed: 09/26/08

$\begin{array}{lllllll}5.03 \mathrm{E}-1 & \mathrm{~N} / \mathrm{A} & \mathrm{ug} / \mathrm{L} & 5.00 \mathrm{E}-1 & \mathrm{ND} & 101 & 75-125\end{array}$


RCRA Metals By PNNL-AGG-415/Water Extract - Quality Control

Environmental Science Laboratory

\begin{tabular}{|c|c|c|c|c|c|c|c|c|c|c|}
\hline & & orting & & Spike & Source & & $\%$ REC & & RPD & \\
\hline Analyte & Result & Limit & Units & Level & Result & $\%$ REC & Limits & RPD & Limit & Notes \\
\hline
\end{tabular}

Batch 8I26002 - 1:1 Water Extract (ICP/ICPMS)

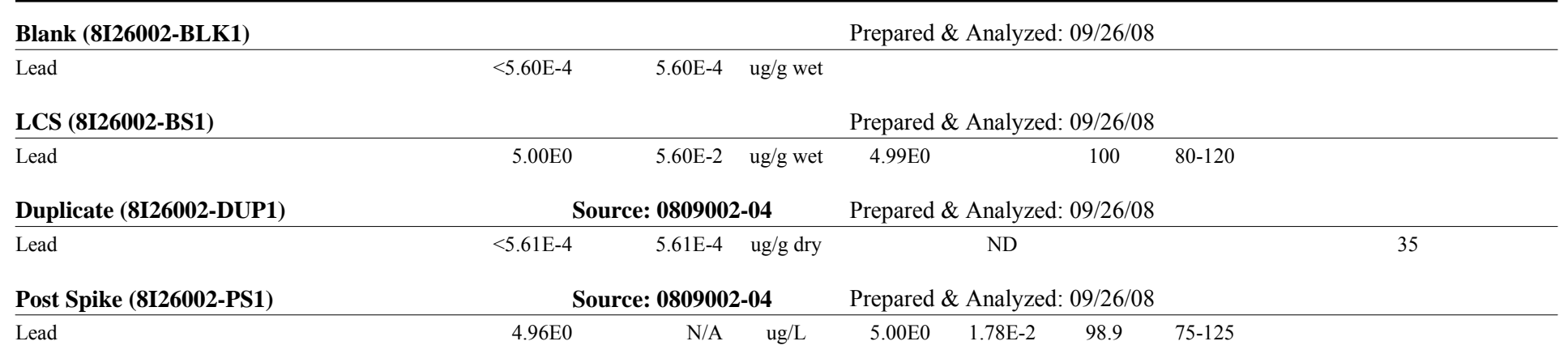




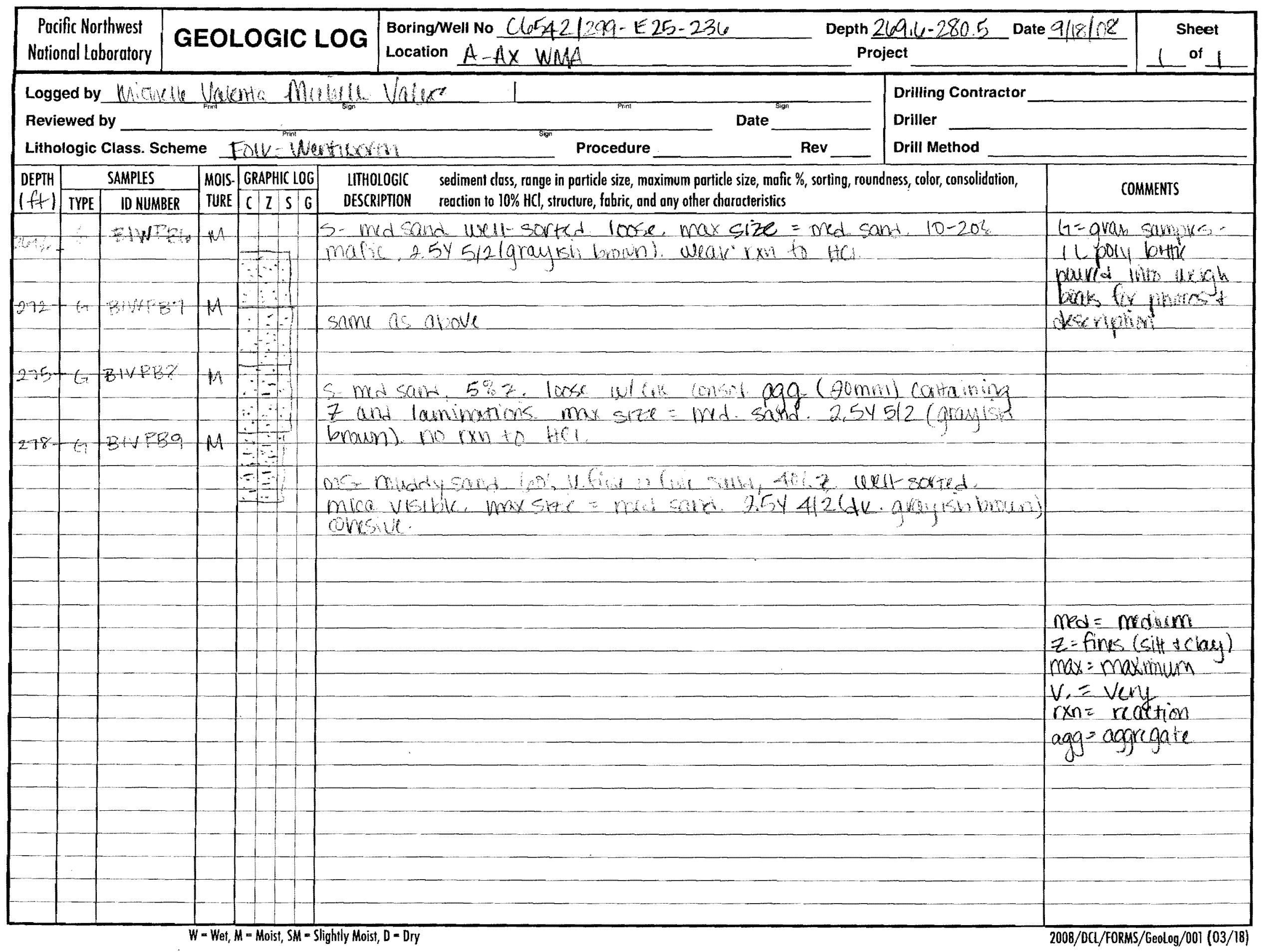




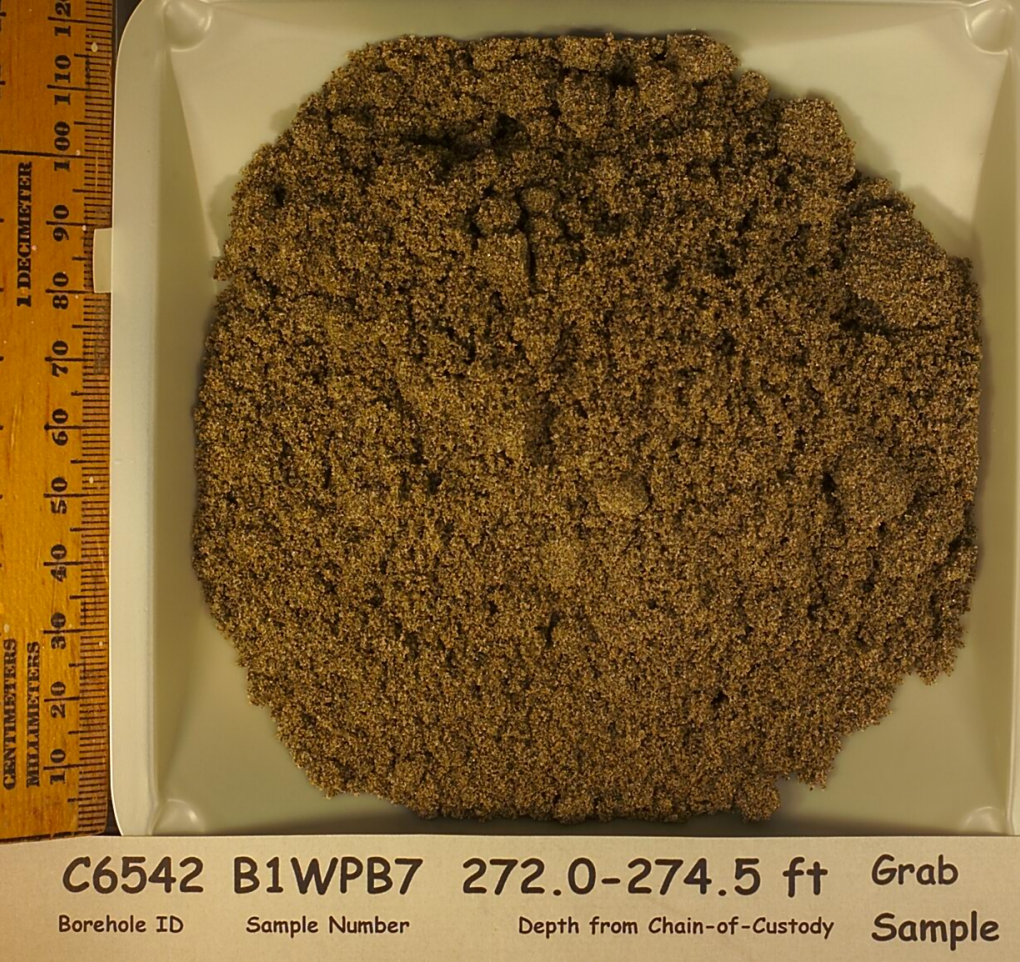




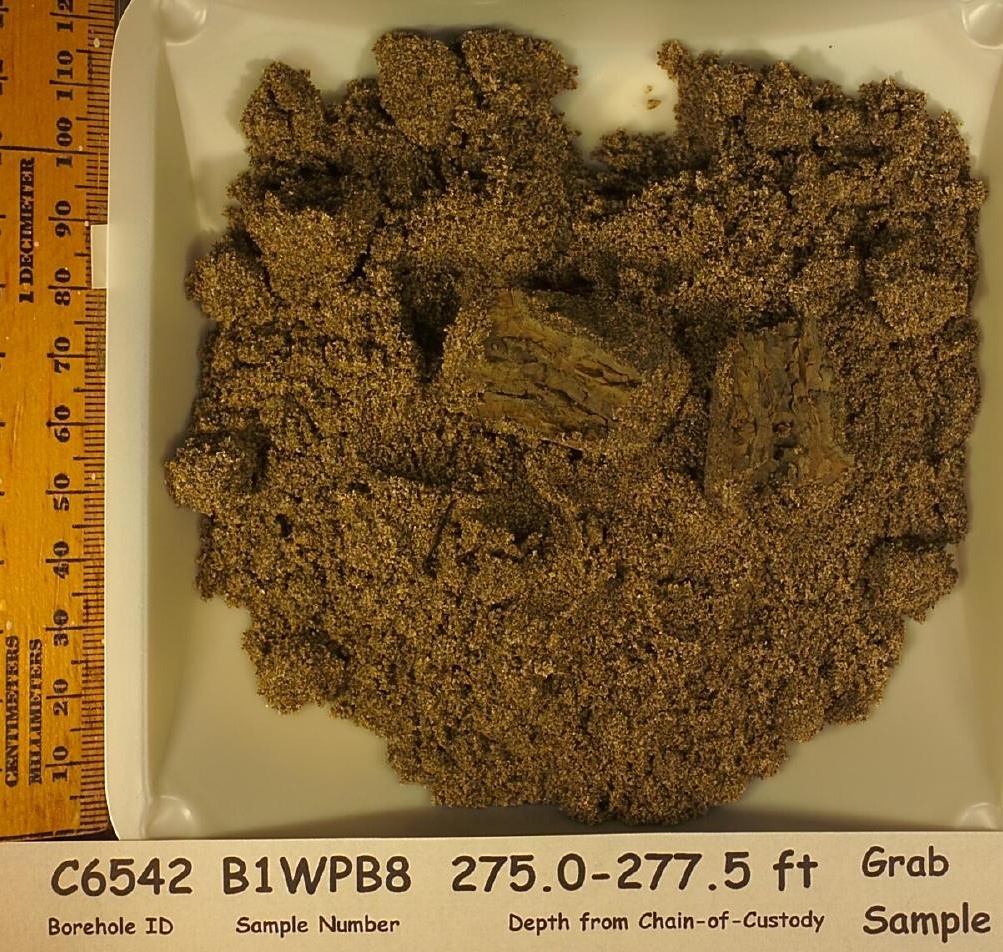




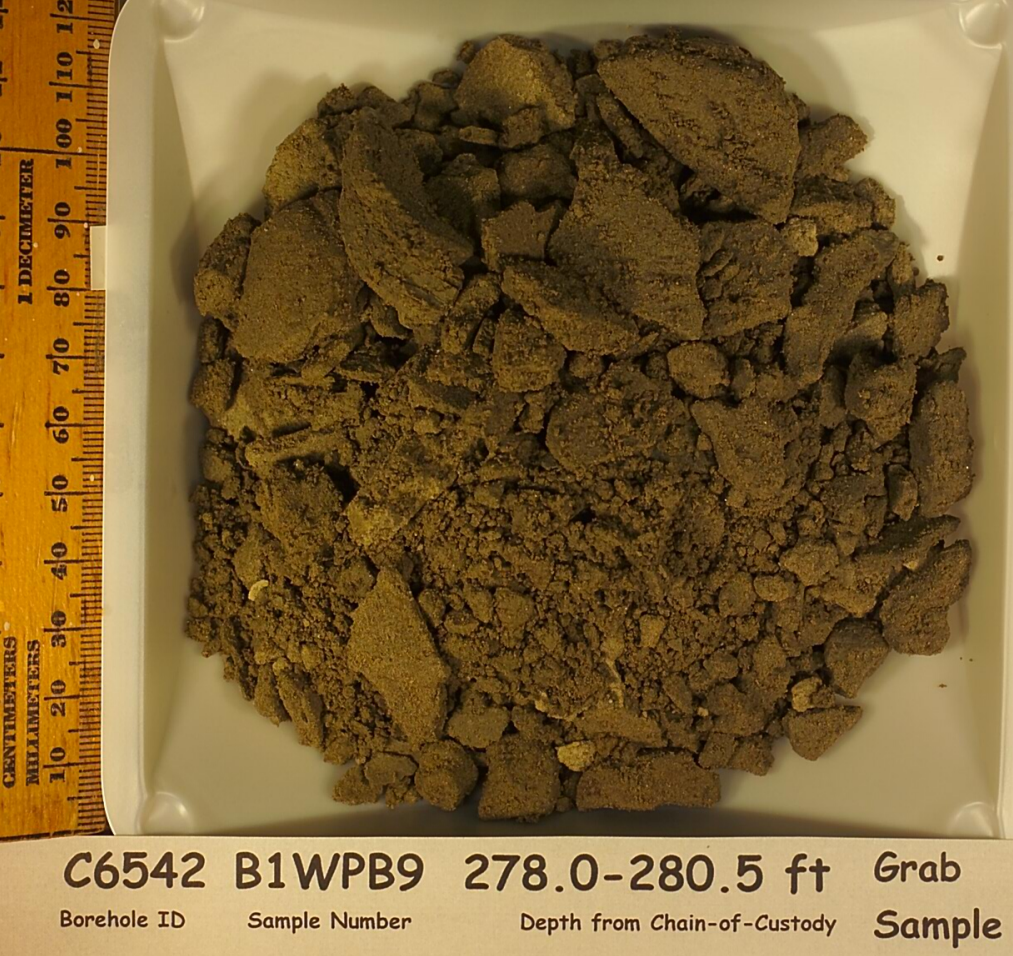




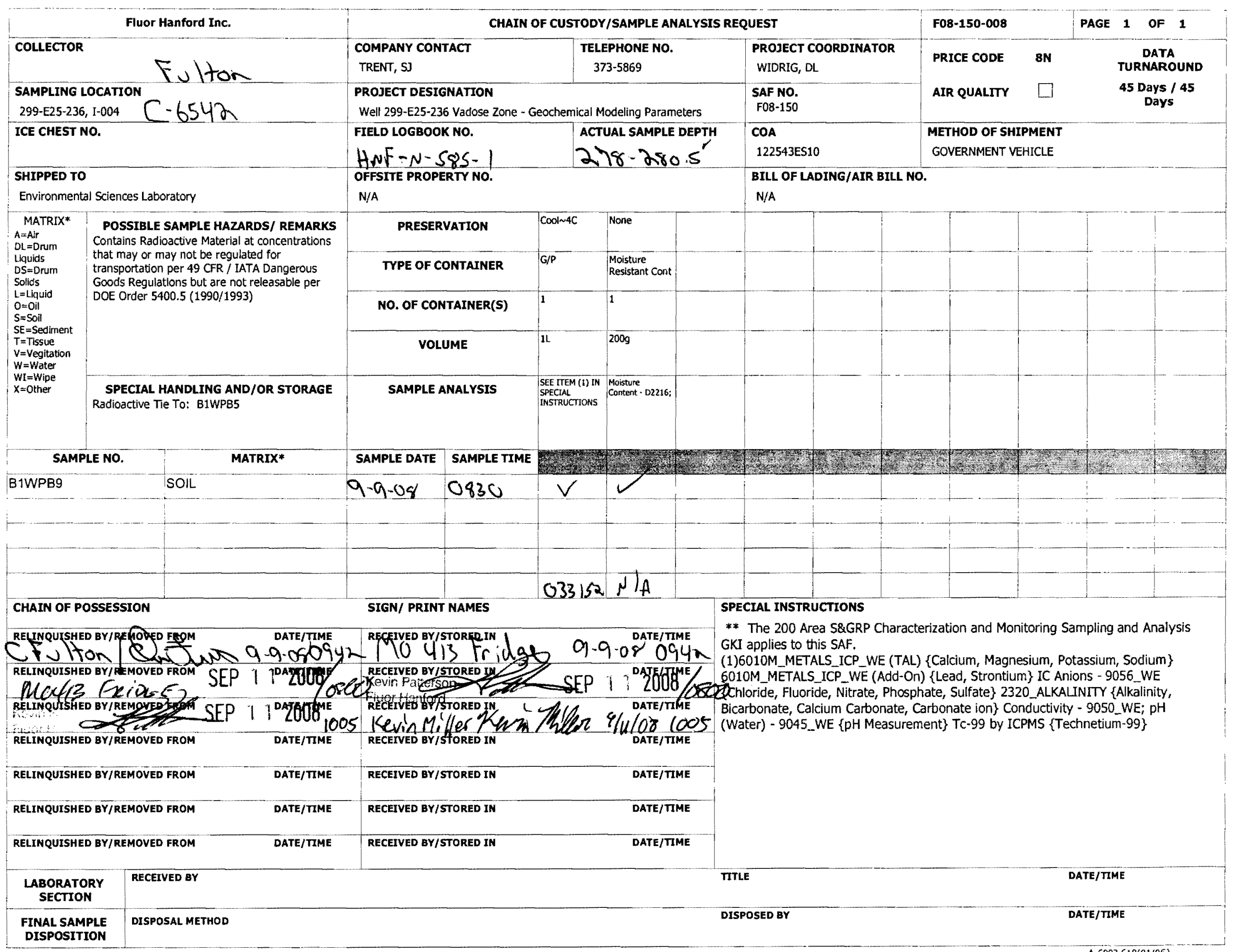

